\title{
Operation Mode of Intelligent Decision Support System Based on Machine Learning
}

\author{
Shan Yuxiang \\ Information Center of China Tobacco Zhejiang Industrial Co., Ltd, Hangzhou, China \\ 188912338@qq.com
}

Keywords: Machine learning, Intelligent decision support system, Applied research

\begin{abstract}
Intelligent decision support system (IDSS) is a new information system, which is formed by introducing artificial intelligence technology into DSS. Knowledge-based reasoning and machine learning technology in artificial intelligence technology is the key to intelligent decision support system, and also the key to the success or failure of intelligent decision support system. This paper first introduces the definition of decision support system, puts forward the combination of artificial intelligence and decision support system, then explains its embodiment and makes comparison. The research on machine self-learning is a hot topic in the field of computer in recent years. The rapid development of machine learning technology provides the realization basis for the composition of a new generation of intelligent decision support system. This paper discusses how to use machine learning method to solve the problems in the research of intelligent decision support system.
\end{abstract}

\section{Introduction}

Intelligent Decision Support System (IDSS) is the product of the combination of Decision Support System (DSS) and artificial intelligence technology. "It introduces the ideas of knowledge representation and knowledge processing in artificial intelligence to decision support systems. Its unique research methods and extensive The emergence of one of the development prospects has become a hotspot and the main development direction of decision support system research [1]. The intelligent decision support system uses the advantages of artificial intelligence and expert system technology in winning analysis and uncertain reasoning, and makes full use of human experience and knowledge in problem solving, providing a new way to solve the above problems [2] By establishing domain expert knowledge base and problem solving subsystem, expert system makes machine intelligence reach or even surpass human expert level in some aspects. The establishment of intelligent decision support system can overcome the limitations of expert system and decision support system, and better support management decision tasks [3]. Artificial intelligence and expert system technology not only have a profound impact on the technology and structure of decision support system, but also on the concept of decision support system. In recent years, many large enterprises and governments are no longer satisfied with simple information processing. With the development and application of artificial intelligence technology, knowledge has become the most important part of information systems [4]. However, in the process of using DSS, users hope that it can be intelligent, so that it can accumulate experience and improve its performance in the process of continuous use.

The essence of decision-making is the thinking process in which decision-makers choose behaviors for decision-making objects according to the information they have [5]. The information provided by this decision is called decision support information. The computer decision support system formed by the combination of computational science and decision science pushes the application of computers in data processing to a new stage of decision support. Decision support system is mainly composed of four parts: database, model library, method library and human-computer interaction. Based on the data of daily business processing system, it uses mathematical or intelligent methods to analyze and synthesize the data, predict the change trend of 
future business, and assist decision makers at all levels to solve semi-structured decision-making problems.

\section{Idss Application System}

\subsection{Present Situation.}

Decision support system is a man-machine interactive information system that uses information technology as a means, applies theories and methods of decision science and related disciplines, and provides help for managers to make correct decisions by providing background materials, assisting in defining problems, revising and improving models, listing possible schemes, and making analysis and comparison, etc. for certain types of decision problems. As a major aspect of information system in management field, intelligent decision support system has become a hot spot and main development direction of computer management application research. Practice shows that only when DSS has rich knowledge and strong knowledge processing ability can it provide more effective decision support to decision makers. For a long time, two key issues in the research of intelligent decision support systems have puzzled researchers of intelligent decision support systems, namely, how intelligent decision support systems acquire and accurately knowledge directly related to the decision process, and how to make intelligent decisions The support system has a certain degree of adaptability and evolution capabilities, allowing it to accumulate experience and improve its own performance in the process of continuous use. Vulnerability and knowledge acquisition difficulties, traditional rule-based methods: acquiring knowledge from experts, sometimes experts know how to do it, but they cannot effectively generalize the rules, the flexibility and adaptability are not enough, and they cannot be formulated according to actual environmental requirements "Dynamic learning strategies" therefore limit the flexibility and adaptability of intelligent assistance [7]. Integration needs to be strengthened: in terms of integration of human-computer intelligence, integration of different software systems, integration of different intelligent technologies, etc. "it is difficult to solve the contradiction between universality and efficiency. In recent years, researchers have proposed a DSS structure composed of knowledge system, language system and problem-solving system, which introduces the problem-solving technology of more mature expert system into the DSS architecture, overcomes the general lack of knowledge in traditional DSS, and plays a great role in promoting, but it does not reflect the initiative of users in model building and model selection, The closed automatic solution process of the framework system also does not have the learning ability, and cannot learn new knowledge and experience accumulation [8]. Therefore, this purely knowledge-based framework system is not suitable for the complicated reality IDSS. A structural form of the intelligent decision support system is shown in figure 1.

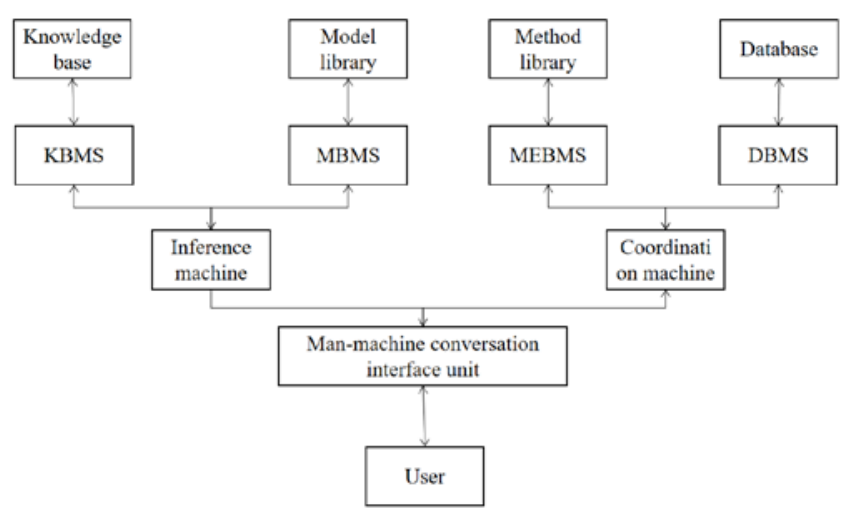

Fig.1 Block Diagram of the Intelligent Decision Support System 


\subsection{Concrete Embodiment and Comparison in Idss}

The effect of learning is closely related to the environment and level of knowledge of the system. The so-called environment can be the object or the objective conditions in which the object is located. The quality of a learning system is closely related to the design of the knowledge system. Knowledge representation and reasoning methods are the key points to consider when designing a learning system. The knowledge in the learning system knowledge base is divided into two types: long-term memory (LTM) and mid-term memory (MTM) [9]. Decision-making: It uses knowledge in the knowledge base to make decisions or take actions to complete various tasks. The environment and knowledge base represent external information sources and learning results respectively, and interact with each other by processing knowledge through decision-making and learning links. Therefore, the environment, knowledge base, learning link and decision-making link constitute the necessary components of the learning system. The intelligence of IDSS is embodied in the ability to provide users with an easy to understand and learn. The human-computer interface with perfect functions generally uses logical paradigm or natural language as the description language of problems, models and knowledge. Another embodiment of intelligence in IDSS is the introduction of knowledge reasoning structure. "By representing the decision-maker's thinking methods and decision experience in a certain way, using reasoning mechanism to simulate the decision-maker's thinking process, guiding the decision-maker to choose the appropriate model by interactive dialogue, rule-based reasoning and other methods. An IDSS should have the ability to decompose tasks according to decision-making problems, automatically select and combine appropriate models for solution, and perform heuristic control in model construction and model operation, which is intelligent scheduling [10]. An IDSS should also have this learning ability \# This is mainly reflected in IDSS's decision-making problem solving process. Can automatically analyze the decision-making strategies of decision-makers during the decision-making process \# Extract decision-making experience and heuristic rules, improve the problem base, and finally automatically extract problem characteristics, obtain problem classification rules and corresponding solution strategies, to assist decision-makers in effective Decision. Intelligent decision support system is a knowledge-based system. Since knowledge management is still a rapidly developing field, it is difficult to give a definitive induction. The above discussion about the types of decision support system is based on the main characteristics of the system, from the perspective of the development of system intelligence, and has the characteristics of several models. Table 1 compares several diss from the aspects of learning ability, intelligent behavior and decision-making method.

Table 1 Comparison of Idss Models

\begin{tabular}{|l|l|l|l|l|l|l|}
\hline Category & $\begin{array}{l}\text { Knowledge } \\
\text { base type } \\
\text { knowledge }\end{array}$ & $\begin{array}{l}\text { Completeness of } \\
\text { knnowledge } \\
\text { ability }\end{array}$ & $\begin{array}{l}\text { Understanding } \\
\text { the decision } \\
\text { process }\end{array}$ & $\begin{array}{l}\text { Main } \\
\text { reasoning }\end{array}$ & $\begin{array}{l}\text { Key Decision } \\
\text { Tools }\end{array}$ \\
\hline $\begin{array}{l}\text { Active } \\
\text { Decision } \\
\text { Support } \\
\text { System }\end{array}$ & Static & $\begin{array}{l}\text { Complete } \\
\text { without conflict }\end{array}$ & No & Have & $\begin{array}{l}\text { Adopt } \\
\text { predefined } \\
\text { process }\end{array}$ & $\begin{array}{l}\text { Models, data, } \\
\text { cognitive } \\
\text { models }\end{array}$ \\
\hline $\begin{array}{l}\text { Decision } \\
\text { Expert } \\
\text { System }\end{array}$ & Static & $\begin{array}{l}\text { Complete } \\
\text { without conflict }\end{array}$ & No & May not & $\begin{array}{l}\text { Deductive } \\
\text { reasoning }\end{array}$ & $\begin{array}{l}\text { Knowledge } \\
\text { base }\end{array}$ \\
\hline $\begin{array}{l}\text { Adaptive } \\
\text { decision } \\
\text { support } \\
\text { system }\end{array}$ & Dynamic & $\begin{array}{l}\text { Allow } \\
\text { incomplete and } \\
\text { conflict }\end{array}$ & Have & Generally have & $\begin{array}{l}\text { Deductive } \\
\text { and inductive } \\
\text { reasoning }\end{array}$ & $\begin{array}{l}\text { Data, intelligent } \\
\text { decision } \\
\text { models, } \\
\text { knowledge } \\
\text { bases }\end{array}$ \\
\hline $\begin{array}{l}\text { Integrated } \\
\text { Decision } \\
\text { Support } \\
\text { System }\end{array}$ & dynamic & $\begin{array}{l}\text { Allow } \\
\text { incomplete and } \\
\text { conflict }\end{array}$ & Have & Have & $\begin{array}{l}\text { Deduction, } \\
\text { induction, } \\
\text { case-based } \\
\text { reasoning }\end{array}$ & $\begin{array}{l}\text { Cognitive } \\
\text { models, } \\
\text { intelligent } \\
\text { computing } \\
\text { methods, } \\
\text { machine }\end{array}$ \\
\hline
\end{tabular}




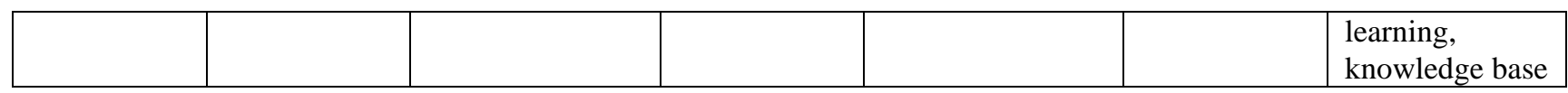

\section{Idss Based on Machine Learning and Its Discussion}

\subsection{Machine Learning Introduces Idss}

Traditional IDSS uses data models and numerical calculation methods to assist decision-making. It has the limitation of not being able to represent complex decision-making processes. With the development of AI technology, it can enable knowledge processing to conveniently and accurately simulate the objective world. Reflect the decision-making process, thereby effectively solving semi-structured and unstructured problems, and forming the initial IDSS. Machine learning is the study of machines that acquire new knowledge and skills, and identify existing knowledge. The "machine" mentioned here refers to a computer, which is now an electronic computer, and may be a seed computer, a photon computer, or a neural computer in the future. By searching the data for statistical patterns and relationships, the records are aggregated into specific classifications to generate rules and rule trees. The advantage of this method is that it can only provide prediction and classification models and can generate clear rules from the data. For example, the commonly used recursive classification algorithm can gradually reduce data subsets. The data is separated into finer subsets to generate decision trees. Decision trees are abstract descriptions of data sets and can be used as knowledge for reasoning. Machine learning can solve the problem of knowledge acquisition bottleneck in expert systems to a certain extent due to its ability to automatically acquire knowledge. Machine learning is one of the earliest topics in artificial intelligence research, and is also one of the most intelligent features and cutting-edge research topics in artificial intelligence. The significant progress in machine learning means that artificial intelligence research has taken a solid step forward. Machine learning has been widely used in natural language understanding, nonmonotone reasoning, machine vision, pattern recognition and other fields, especially in knowledge systems and decision science. Although the research of machine learning in the field of IDSS has just started, just as database systems and expert systems have had a huge impact on IDSS in the past ten years, machine learning will play a role in fueling the new generation of research. If the previous IDSS research focuses on the processing of data and knowledge, then machine learning is a study of IDSS at the strategic level. If IDSS has a certain learning ability, it can solve useful problems under the guidance of domain knowledge. Knowledge is stored in an appropriate way for future use, enabling IDSS to self-improve in applications.

\subsection{Research on the Application of Machine Learning in Idss Needs Further Exploration}

The problem recognition function of language subsystem and problem processing subsystem in IDSS is a problem of natural language understanding, which means that the computer system extracts its semantics from the natural language request input by the user. IDSS can use the concept similar to "user model" in intelligent guidance system to obtain the preference of decision makers. According to the user's response to a series of problems, the intelligent guidance system decides what kind of difficulty and level information to provide to the user. Therefore, IDSs learning components learn by observing the past performance of users, and can also learn lessons from previous wrong decisions as experience preservation. Improve the existing learning algorithm, make full use of the characteristics of various learning algorithms. IDSS often involves a lot of data processing, so it is very important to overcome the shortcomings of the above induction algorithm to improve the working environment of IDSS, because IDSS needs to strengthen the ability to express and deal with the common uncertainties in most decision support tasks. The application of machine learning in IDSS still has many problems to be further studied, such as the exploration of effective learning algorithms, neural network, fuzzy logic, genetic algorithm, rough set theory, etc. are also widely used in machine learning. IDSS must be able to use different methods to solve different decision-making problems according to their characteristics. This heavy task can be simplified by learning methods to 
accumulate solution experience and learning solution steps. Among them, the further contents to be discussed include deepening understanding of the computational characteristics of inductive learning, applying machine learning to improve the user section of intelligent decision support system, developing adaptive intelligent decision support system and enhancing group decision support through machine learning.

\subsection{Application of Reinforcement Learning in Idss Based on Agent Technology}

Reinforcement learning is the learning of intelligent system from environment to behavior mapping, so as to maximize the value of reinforcement signal function. Because the external environment provides little information, IDSs must learn from its own experience. Reinforcement learning is an important machine learning method, a calculation method based on understanding, and also an intentional automatic learning and decision-making process. Compared with other calculation methods, it is significantly different in that it emphasizes the interactive learning between individuals and the environment, and does not rely on the typical external supervision and the complete model of the environment. Agent is a hot research topic in the field of pre-oral AI, mainly including intelligent Agent research, Multi-Agent system research and Agent-oriented programming research. knowledge, oral label and ability are the three elements of Agent itself. Agent technology makes it a reality to establish a decision support system that realizes network, intelligence and integration of man-machine coordination. Therefore, it is of great significance to study the new generation IDSS model based on MAS technology. The application of reinforcement learning in IDSS based on Agent technology will also be paid attention to. Agent is mainly divided into the following aspects: 1 . Interface Agent is an organic whole composed of human and computer through human-computer interface. It replaces the traditional human-computer interaction interface and emphasizes the autonomy and learning of Agent. 2. Information Agent is a kind of agent used for information retrieval. In intelligent decision support system, information agent can be used to realize decision information retrieval and scheduling. 3. Mobile Agent means to move freely in a complex network system, and to transfer the operation results back through the network. 4. Collaboration Agent includes various collaboration protocols, strategies, processing and evaluation of collaboration. The aim is to find a strategy that can be accepted by both parties of the collaboration. Collaborative processing refers to the specific behaviors of the agents in the process of collaboration. Collaborative evaluation is to evaluate the information received and the collaboration methods adopted.

\section{Conclusions}

In this paper, we first study and discuss the related technologies of machine learning in IDSS, and deeply discuss the technical problems, as well as the new IDSS based on reinforcement learning and statistical machine learning in the theory, design and construction of IDSS based on machine learning. Intelligence and decision support are two characteristics of IDSS today. With the development of new technologies in AI field, it is one of the most important issues for IDSS to combine the advantages of machine learning into the construction of IDSS. With the continuous development and in-depth research of decision-making technology and computer technology, the research of intelligent decision support technology based on machine learning is bound to have broad application prospects and has important theoretical value and practical significance.

\section{References}

[1] Li Yanan, Ni Hongmei, Fang Shengquan, \& Yang Xuming. (2016). Research on Intelligent Decision Support System for Ebola Epidemic Prevention and Control Based on Internet. Medical Information, 29 (8), 7-8.

[2] Wang Juan, \& Li Rui. (2015). Research on Multi-agent Based Power System Information Security Decision Support System. Electric Power Information and Communication Technology (3), 82-87. 
[3] Huang Guangbin. (2017). Intelligent Revolution: Synergy of Artificial Intelligence, Machine Learning, Biological Learning and Intelligent Materials. Software and Integrated Circuits (4), 18-24.

[4] Yang Pan. (2016). Research on Machine Learning Based on Artificial Intelligence. Digital Technology and Application (11), 89-89.

[5] Zhang Weiyan, Zhai Qian, \& Xiangming Ming. (2019). Research Status of Anesthesia and Neural Circuits and Artificial Intelligence. International Journal of Anesthesiology and Resuscitation, 40 (1), 81-85.

[6] Lin Yongqing. (2017). Intelligence is Algorithm: Ultimate Algorithm and Machine Learning. Financial Expo (6), 44-45.

[7] Zhu Chong, Chen Xuefei, \& Zhang Congpin. (2015). Advances in Machine Learning Research. Fujian Computer (8), 70-70.

[8] Meng Yu. (2019). Machine learning makes computers smarter. Computers and Networks (14), 13-13.

[9] Jiang Luning. (2016). Machine learning, deep learning and network security technology. China Information Security (5), 94-94.

[10] Jianjian Ma. (2015). Research on Machine Learning Methods and Applications. Computer Knowledge and Technology, 11 (19). 